\title{
Genome-wide association mapping of resistance against rice blast strains in South China and identification of a new Pik allele
}

\author{
Chenggang Li ${ }^{1}$, Dan Wang ${ }^{2}$, Shasha Peng ${ }^{2}$, Yue Chen ${ }^{1}$, Pin Su', Jianbin Chen ${ }^{1}$, Limin Zheng ${ }^{1}$, Xinqiu Tan', \\ Jinling Liü ${ }^{2}$ Yinghui Xiao ${ }^{2}$, Houxiang Kang ${ }^{3}$, Deyong Zhang ${ }^{1 *}$, Guo-Liang Wang ${ }^{2,4^{*}}$ (D) and Yong Liu ${ }^{1^{*}}$
}

\begin{abstract}
Background: Effective management of rice blast, caused by the fungus Magnaporthe oryzae, requires an understanding of the genetic architecture of the resistance to the disease in rice. Rice resistance varies with $M$. oryzae strains, and many quantitative trait loci (QTLs) affecting rice blast resistance have been mapped using different strains of $M$. oryzae from different areas. However, little is known about the genetic architecture of rice resistance against the $M$. oryzae population in Hunan Province, which is a main rice production area in South China.

Results: In this study, we used three isolates from Hunan Province and the rice diversity panel 1 to perform a genome-wide association study (GWAS) of blast resistance in rice. A total of 56 QTLs were identified. One of the QTLs is localized with the resistance gene Pik locus which confers resistance to all three isolates. Genomic sequence analysis of the resistant cultivars led to the identification of a new Pik allele, which we named Pikx. Yeast two-hybrid and co-immunoprecipitation assays between AvrPiks and Pikx confirmed that Pikx is a new allele at the Pik locus.

Conclusions: Our GWAS has identified many new blast resistance QTLs. The identified new Pik allele Pikx will be useful for breeding cultivars with high resistance to blast in Hunan and other South China provinces. Further research on the relationship between AvrPiks and Pikx will provide new insights into the molecular mechanism of rice resistance to $M$. oryzae.
\end{abstract}

Keywords: GWAS, Rice blast, QTL, R gene, Pikx

\section{Background}

Rice is a staple food of more than half of all people worldwide (Gnanamanickam 2009). However, rice production is affected by many diseases that threaten the food security of the increasing world population. Rice blast, caused by the fungal pathogen Magnaporthe oryzae, is a destructive disease of rice (Valent and Chumley 1991). It typically causes an annual yield loss of $10-30 \%$ and leads to large economic losses in many countries (Skamnioti and Gurr

\footnotetext{
*Correspondence: dyzhang78@163.com; wang.620@osu.edu;

haoasliu@163.com

${ }^{1}$ State Key Laboratory of Hybrid Rice and Institute of Plant Protection, Hunan Academy of Agricultural Sciences, Changsha 410125, China

${ }^{2}$ Southern Regional Collaborative Innovation Center for Grain and Oil Crops in China and College of Agronomy, Hunan Agricultural University, Changsha 410128, Hunan, China

Full list of author information is available at the end of the article
}

2009). Rice varieties with blast resistance can help control this pathogen (Hulbert et al. 2001).

Two types of resistance genes are responsible for rice blast resistance: major resistance $(R)$ genes that confer race-specific resistance and quantitative trait loci (QTLs) that control partial, nonrace-specific resistance (Skamnioti and Gurr 2009). More than 100 blast resistance loci or genes have been mapped to rice chromosomes (Fang et al. 2016). Among these, only $28 R$ genes and 2 QTLs have been cloned and characterized (Xiao et al. 2017; Deng et al. 2017; Zhao et al. 2018). These cloned R genes are distributed across all 12 chromosomes except chromosome 3 (Yang et al. 2009). All of the cloned $R$ genes except for Pi-d2, pi21 and Ptr contain nucleotidebinding domain leucine-rich repeat (NLR) proteins (Liu et al. 2010; Zhao et al. 2018). 
Among the cloned $R$ genes, the Pik locus is especially important because it harbors a number of blast $R$ genes used in rice breeding (Zhai et al. 2011). The Pik locus has at least six alleles (Pik, Pikm, Pikp, Piks, Pikh, and Pi1) that cluster on the end of the long arm of chromosome 11 (Zhai et al. 2011; Liu et al. 2014). Three of these alleles (Pikm, Pikp, and Pik) have been isolated and characterized (Zhai et al. 2011). Because $R$ genes are highly specific to $M$. oryzae races, resistance of a single $R$ gene is often rapidly overcome by the selection of compatible pathogen races (Hittalmani et al. 2000; Oliveira-Garcia and Valent 2015). In response to the rapid evolution of $M$. oryzae, the rice genome has evolved $R$ gene polymorphism, which confers multiple forms of race-specific resistance (Hayashi et al. 2004). For example, the physical interaction of Pik alleles with specific $A v r-P i k$ alleles can be explained by the coevolution of pathogen and host (Yoshida et al. 2009; Kanzaki et al. 2012; Wu et al. 2014). The Pik gene comprises two NBS-LRR genes, $P i k-1$ and $P i k-2$, and the former acts as the senor for the interaction with the corresponding AvrPik protein and the latter is responsible for defense activation and signaling. A recent study showed that polymorphic residues in Pik-1 determine the resistance specificity (Carlos et al. 2018)

Genome-wide association study (GWAS) has recently been used for assessing associations between genetic markers and blast resistance in rice. GWAS was first used to identify genes underlying complex diseases in humans (Altshuler et al. 2008). With its wider use and the development of GWAS modeling (Price et al. 2006; $\mathrm{Yu}$ et al. 2006; Liu et al. 2016), GWAS has become a powerful approach for mapping a number of traits of rice, including agronomic traits (Huang et al. 2010; Zhao et al. 2011) and tolerance to abiotic stress (Zhu et al. 2015; Lv et al. 2016; Wang et al. 2016a, 2016b; Kaler et al. 2017). In rice, GWAS in combination with highthroughput sequencing and gene knockout techniques has been used to rapidly identify new functional genes that influence yield, heading, awn length, and other agronomic traits (Si et al. 2016; Yano et al. 2016).

In their study of resistance to 16 representative blast strains collected from all over China, researchers recently identified 30 loci associated with blast resistance using an indica rice population (Wang et al. 2014). The rice diversity panel 1 (RDP1), which comprises over 400 rice cultivars from 82 countries, was developed for GWAS of agronomical traits, and is publicly available (Zhao et al. 2011). Using RDP1, our team previously performed an association study of rice resistance to blast isolates from Asia (China, India, the Philippines, and South Korea) and the Americas (Colombia), which led to the identification of 97 loci associated with blast resistance along with two new Pi5 alleles (Kang et al. 2016). Using eight $M$. oryzae isolates from four African counties, 31 rice genomic regions associated with blast resistance were identified on another study (Mgonja et al. 2016). Similarly, our team analyzed QTLs associated with blast resistance in the field and identified 16 loci associated with field blast resistance using RDP1 (Zhu et al. 2016).

In the current study, we performed GWAS on rice blast using the 234 RDP1 cultivars and three isolates of the pathogen from the major rice production regions in Hunan Province, China. A total of 56 QTLs associated with blast resistance were identified in the rice genome. Only one QTL associated with resistance to all three isolates was found, and it was localized with the known $R$ gene Pik locus. Genotype analysis of the significantly associated SNP-11.27701887 on the Pik locus in 234 cultivars confirmed the association between genotype and resistance to the three isolates. In addition, sequence analysis and protein-protein interaction analysis indicated that this gene is a previously unreported allele on the Pik locus. The new allele was named Pikx.

\section{Methods \\ Plant and fungal materials}

A total of 234 rice accessions from RDP1 were used, and these comprised 59 tropic japonica (TRJ), 35 temperate japonica (TEJ), 53 indica (IND), 45 aus (AUS), 10 aromatic (ARO), and 32 admixture (ADMIX) accessions (Zhao et al. 2011). Fifteen seeds of each accession were germinated and sowed in small pots with soil. The rice seedlings were grown in a growth chamber under controlled conditions $\left(26^{\circ} \mathrm{C}, 75 \%\right.$ relative humidity, and a $10 \mathrm{~h}$ light $/ 14 \mathrm{~h}$ dark photoperiod). Three $M$. oryzae isolates, 110-2, 193-1-1, and 87-4, were collected in Dong'an County, Taojiang County, and Hanshou County, respectively, in Hunan Province (Fig. 1e). The M. oryzae isolates were cultured on an oat medium to obtain conidia for inoculation of 3-week-old rice seedlings.

\section{Blast resistance phenotyping of rice cultivars}

$M$. oryzae conidia at a spore concentration of $1 \times 10^{5}$ were sprayed onto rice leaves as described by Park et al. (2012). Each cultivar was inoculated with the three isolates in three replications. Approximately 7 days after inoculation with $M$. oryzae, disease was scored on a scale from 0 to 9 based on the size and area ratio of lesions as described previously (Kang et al. 2016). The disease score for each pot was measured, and the average values of the three replications were used to generate a data matrix.

\section{GWAS of rice blast resistance}

The 277,524 SNP genotypes of the 234 RDP1 accessions were generated from the $700 \mathrm{~K}$ SNP RDP1 genotypes using P-link with the criterion of minor allele frequency $\geq$ $5 \%$. GWAS was performed with the software EMMAX 
A

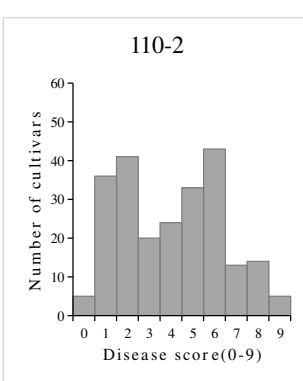

B

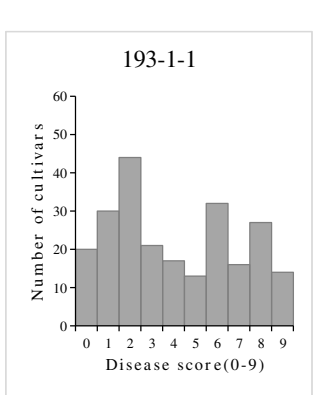

C

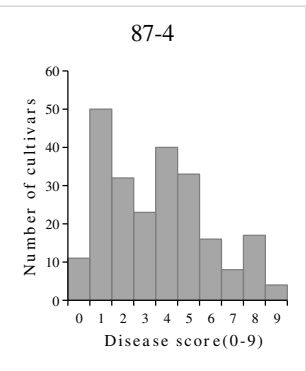

D

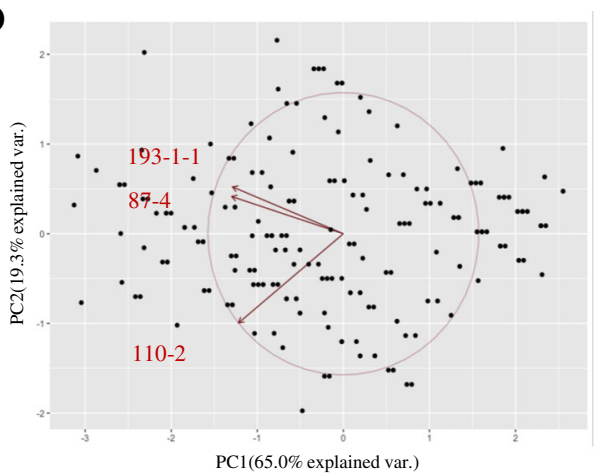

E

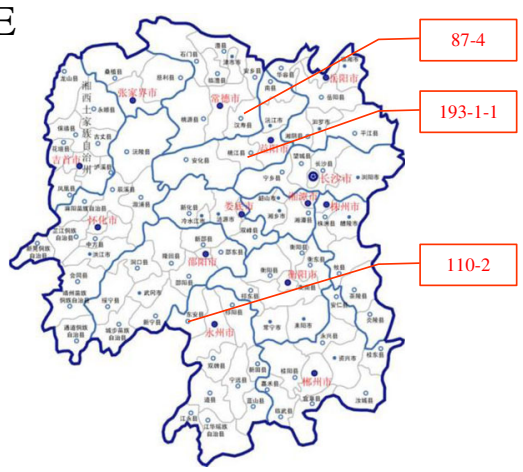

Fig. 1 The information of three M. oryzae isolates and blast disease scores of the 234 RDP1 cultivars. a-c The distribution of disease scores of the 234 RDP1 cultivars against isolates 110-2, 193-1-1 and 87-4. d PCA of resistance phenotypes of the 234 RDP1 cultivars to the three isolates. e Geographic distribution of three M. oryzae isolates in Hunan Province Map

(Kang et al. 2010, http://csg.sph.umich.edu/kang/emmax/) using the 277,524 SNP genotypes and the phenotype dataset. SNPs with $-\log _{10}(P$-value $) \geq 4.0$ were considered to have significant associations. Manhattan and Q-Q plots were generated with the $\mathrm{R}$ environment (https://cran.rproject.org/web/packages/qqman).

\section{Identification of QTLs and selection of candidate genes}

QTLs were identified by using the Nipponbare genome as a reference, and candidate genes from the $200 \mathrm{~kb}$ interval regions around the peak SNPs were selected. All of the reported $\mathrm{R}$ or defense-related genes in plants, including NLR, serine-threonine kinase, and transcription factor, were considered for selection of candidate genes (Liu et al. 2014; Li et al. 2017).

\section{Characterization and validation of the functions of candidate genes}

The association between QTL50 and blast resistance was confirmed based on the analysis of the genotype of the significantly associated SNPs in the 234 cultivars. The Pik alleles in rice and AvrPik alleles in M. oryzae were amplified using the Pik or AvrPik specific primers, respectively, (Additional file 1: Table S1) and the PCR products were sequenced.

The yeast-two hybrid ( $\mathrm{Y} 2 \mathrm{H})$ system and co-immunoprecipitation (Co-IP) assay were used to confirm the interaction between the $\mathrm{R}$ and Avr proteins. The protocols followed those of the Clontech Handbook for Y2H and the Co-IP method previously described Wang et al. (2016a, 2016b). The signal peptide-truncated cDNA fragments of AvrPik-A, $-C,-D$, and $-E$ were synthesized by Genereate Ltd. and were then inserted into the pGBKT7 (for $\mathrm{Y} 2 \mathrm{H}$ ) vector and into the pYBA1152 (for Co-IP) vector. Fragments of Pikx-1$c c$ that were amplified from the cDNA of rice seedlings of cultivar NSFTV_131 using PrimeSTAR GXL DNA polymerase (Takara) were cloned into pGADT7 (for $\mathrm{Y} 2 \mathrm{H}$ ) and PYBA1144 (for Co-IP) using specific primers (Additional file 1: Table S1).

\section{Results}

Resistance of the 234 RDP1 cultivars to the three $M$. oryzae isolates

The distribution of blast disease scores of the 234 cultivars inoculated with the three $M$. oryzae isolates is shown in Fig. 1a, b, and c. Among the inoculated rice cultivars, 11 were highly resistant (disease scores $\leq 1$ ), and 14 were highly susceptible (disease scores $\geq 8$ ) to all the three isolates (highlighted with yellow and blue, respectively, in Additional file 2: Table S2). Principal component analysis (PCA) showed that the levels of resistance of the cultivars to the three isolates were diverse (Fig. 1d). However, the disease scores of the cultivars to isolates 193-1-1 and 874 were clustered perhaps because the two isolates were 
collected in fields that were near each other and that might therefore contain similar avirulence genes.

\section{Association mapping of rice QTLs linked to resistance to M. oryzae}

To identify genomic regions that are associated with blast resistance to the three isolates, we performed a GWAS using the disease scores and the $700 \mathrm{~K}$ SNP genotypes of the inoculated cultivars. A total of 56 QTLs associated with blast resistance to the three isolates were detected in the rice genome ( $-\log _{10} P \geq 4.0$ ) (Fig. 2). Among the QTLs, 24 loci were associated with resistance to isolate $110-2,32$ were associated with resistance to isolate $87-4$, and 22 were associated with resistance to isolate 193-1-1 (Additional file 3: Table S3). Some of the loci were associated with resistance to two isolates, i.e., three loci were associated with resistance to both $110-2$ and $87-4$, two loci to both $110-2$ and 193-1-1, and one locus to 87-4 and 193-1-1. Among all of the mapped QTLs, 16 were located at sites of five previously cloned resistance genes, and 40 were loci identified for the first time in this study. Interestingly, seven QTLs (46-52) were located in the Pik region on chromosome 11 (Fjellstrom et al. 2004) (Additional file 3: Table S3). Among them, QTL48, QTL50, and QTL51 were associated with resistance to all three isolates. In addition, seven of the QTLs (QTL21, QTL48, QTL49, QTL50, QTL51, QTL52 and
QTL53) were also identified in a previous study (Kang et al. 2016) (Additional file 4: Table S4). In particular, the Pik locus was identified in both Kang et al. (2016) and the present study.

\section{QTL50 is linked to the Pik locus}

To determine the relationship between QTL48/QTL50/ QTL51 and Pik, we identified 14 significant SNPs that were linked to the three QTLs (Additional file 3: Table S3) in the 200-kb region. Among them, SNP-11.27606211 and SNP-11.27701887 at QTL50 were tightly associated with resistance to the three isolates (Fig. 3a). Haplotype analysis of the 234 RDP1 cultivars indicated that SNP-11.27701887 had a strong association with the resistance to the three isolates (Fig. 3b). In addition, we sequenced the AvrPik genes from the three isolates using AvrPik-specific primers (Additional file 1: Table S1). Sequence analysis showed that 110-2 and 87-4 harbor AvrPik-D, 193-1-1 harbors Avr gene similar to AvrPik-D and AvrPik-E (Fig. 3c, d), suggesting that QTL50 may be an allele at the Pik gene that can recognize the three AvrPik genes in the fungus.

\section{Identification and validation of the new allele at the Pik locus}

To determine whether the gene at QTL50 is a new allele of Pik, we sequenced 11 blast-resistant rice cultivars

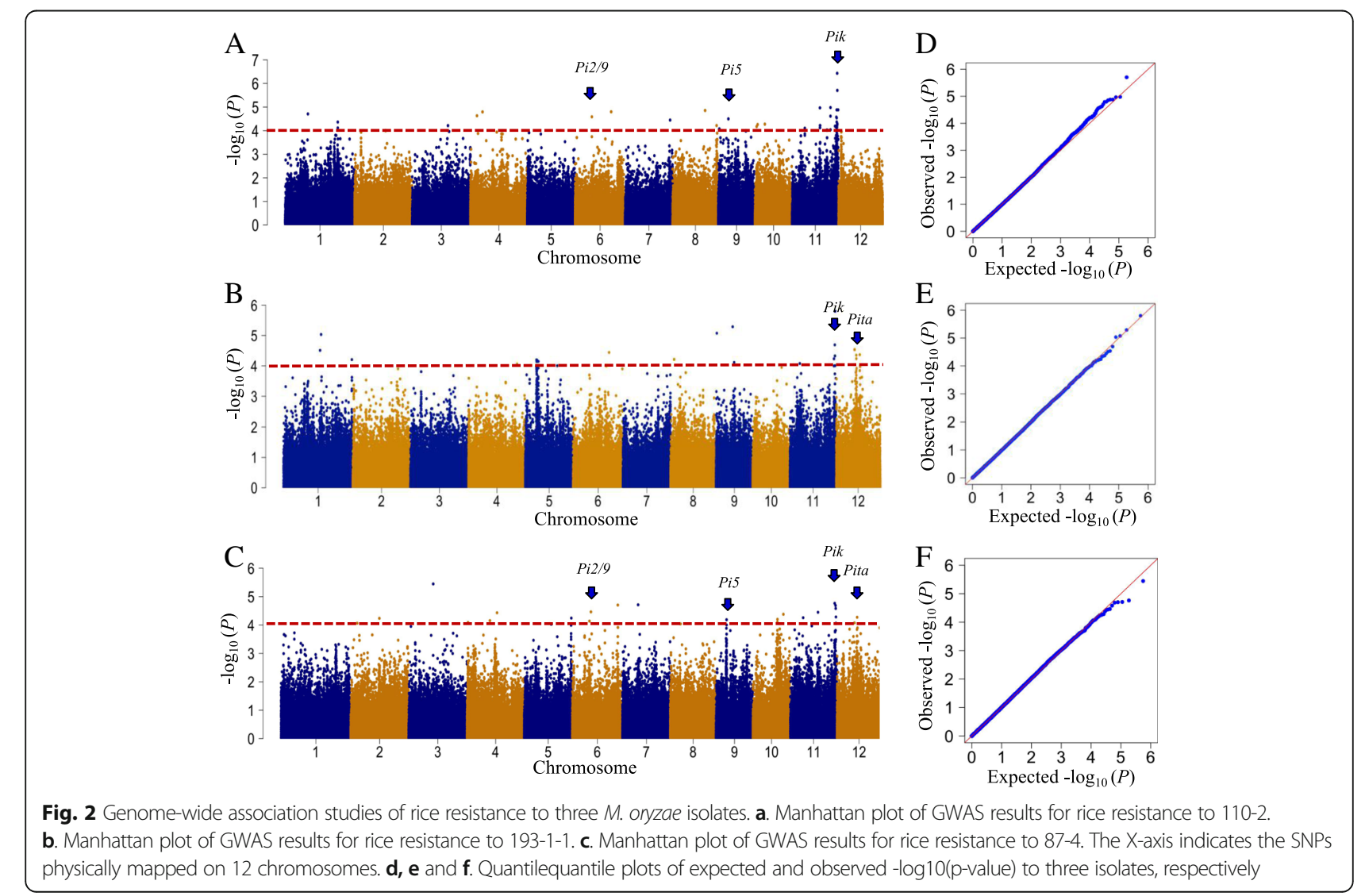


A

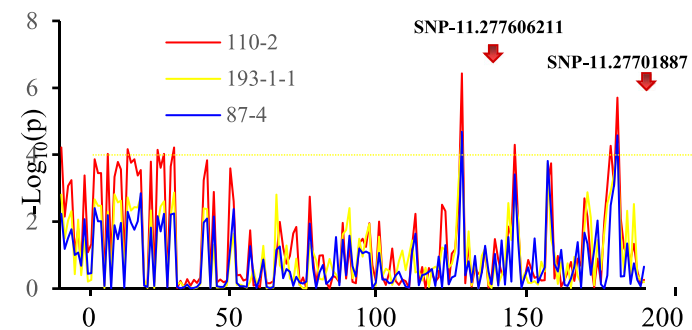

B SNP-11.27701887 alleles

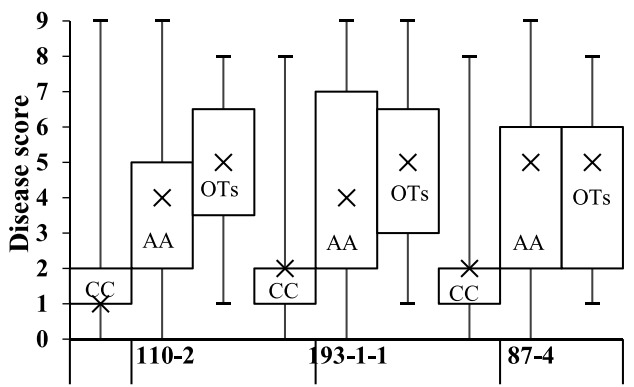

C.

AvrPik-A AvrPik-B AvrPik-C AvrPik-D AvrPik-E AvrPik-110-2 AvrPik-193-1 AvrPik-87-4
40

\begin{tabular}{|c|c|c|c|}
\hline & & & \\
\hline F L & L T L G T V A V VN & A E T G N K Y I E K & R A I D L S R E R D \\
\hline R V T T F N T F L & L T L G T V A V N & A E T G N K Y E K & R A I D L S R E R D \\
\hline M R V T T F N T F L & $\mathrm{L} T \mathrm{~L}$ G T VA V V N & A E T G N K Y I E K & R A I D L S R E R D \\
\hline M R V T T F N T F L & $\mathrm{L} T \mathrm{~T}$ G T VA V V N & A E T G N K Y E K & R A I D L S R E R D \\
\hline M R V T T F N T F & L T L G T V A V N & A E T G N K Y E K & R A I D L S R E R D \\
\hline M R V T T F N T F L & L T L G T V A V VN & A E T G N K Y E K & R A I D L S R E R D \\
\hline M R V T T F N T F L & L T L G T VA V V N & A E T G N Y I E K & R A I D L S R E R D \\
\hline MRVTTFNTF & $\mathrm{L} T \mathrm{~T} \mid \mathrm{G} \mathrm{T} \mathrm{VA} \mathrm{V} \mathrm{VN}$ & A E T G N K Y E & R A I D L S R E R I \\
\hline
\end{tabular}

60

70

80

AvrPik-A

AvrPik-B

AvrPik-C

AvrPik-D

AvrPik-E

AvrPik-110-2

AvrPik-193-1

AvrPik-87-4

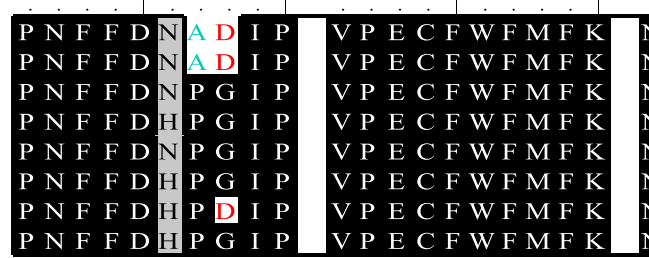

N N V R Q D A G T

N N V R Q D A T

N N V R Q D DG T C

N N V R Q A G T C

N N V R Q D A G T C

$N$ N R Q D G T C

N N V R Q D A G T C

N N R Q D A G T C

90

100

110

AvrPik-A

AvrPik-B

AvrPik-C

G P N W VH I K S D D N C N L S G D F P

AvrPik-D

AvrPik-E

AvrPik-110-2

AvrPik-193-1

AvrPik-87-4

G P NWVH I K S D

G P NWVH I K S D

G P NWVH I K S D

G P NWVH I K S D

G P NWVH I K S D

G P N W VH I K S D

D N C N L S G D F P

D N C N L S G D F P

D N C N L S G D F P

D N C N L S G D F P

D N C N L S G D F P

D N C N L S G D F P

$\begin{array}{lllllll}\text { P G W I V L G K K R } & \text { P G F } & * \\ \text { P G W I VL G K K R } & \text { P G F } & * \\ \text { P G W I V L G K K R } & \text { P G F } & * \\ \text { P G W I V L G K K R } & \text { P G F } & * \\ \text { P G W I V L G K K R } & \text { P G F } & * \\ \text { P G W I V L G K K R } & \text { P G F } & * \\ \text { P G W I V L G K K R } & \text { P G F } & * \\ \text { P G W I V L G K K R } & \text { P G F } & *\end{array}$

D

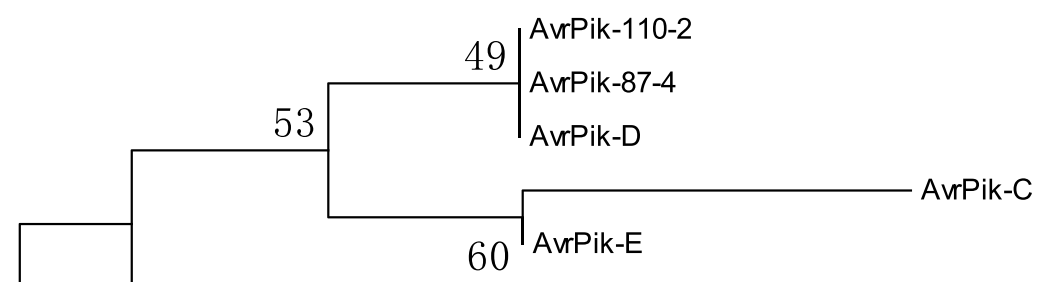

AvPik-193-1-1

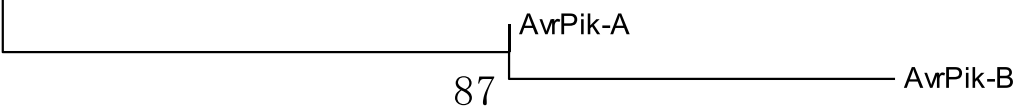

0.002

Fig. 3 Relationship between QTL50 and Pik and the AvrPik sequences in the three isolates a SNP-11.27701887 is the most significant SNP that is associated with resistance to all three M. oryze isolates in QTL50. b SNP-11.27701887 alleles distribution in the 234 RDP1 cultivars. CC, AA and OTs represent the genotype AA, CC and other type of SNP-11.27701887, respectively. c Sequence alignment of the AvrPik amino acid sequences using ClustalW. Shading fonts indicate common amino acid residues in the alleles. $\mathbf{d}$ Phylogeny tree of the AvrPik genes from three M. oryze isolates 


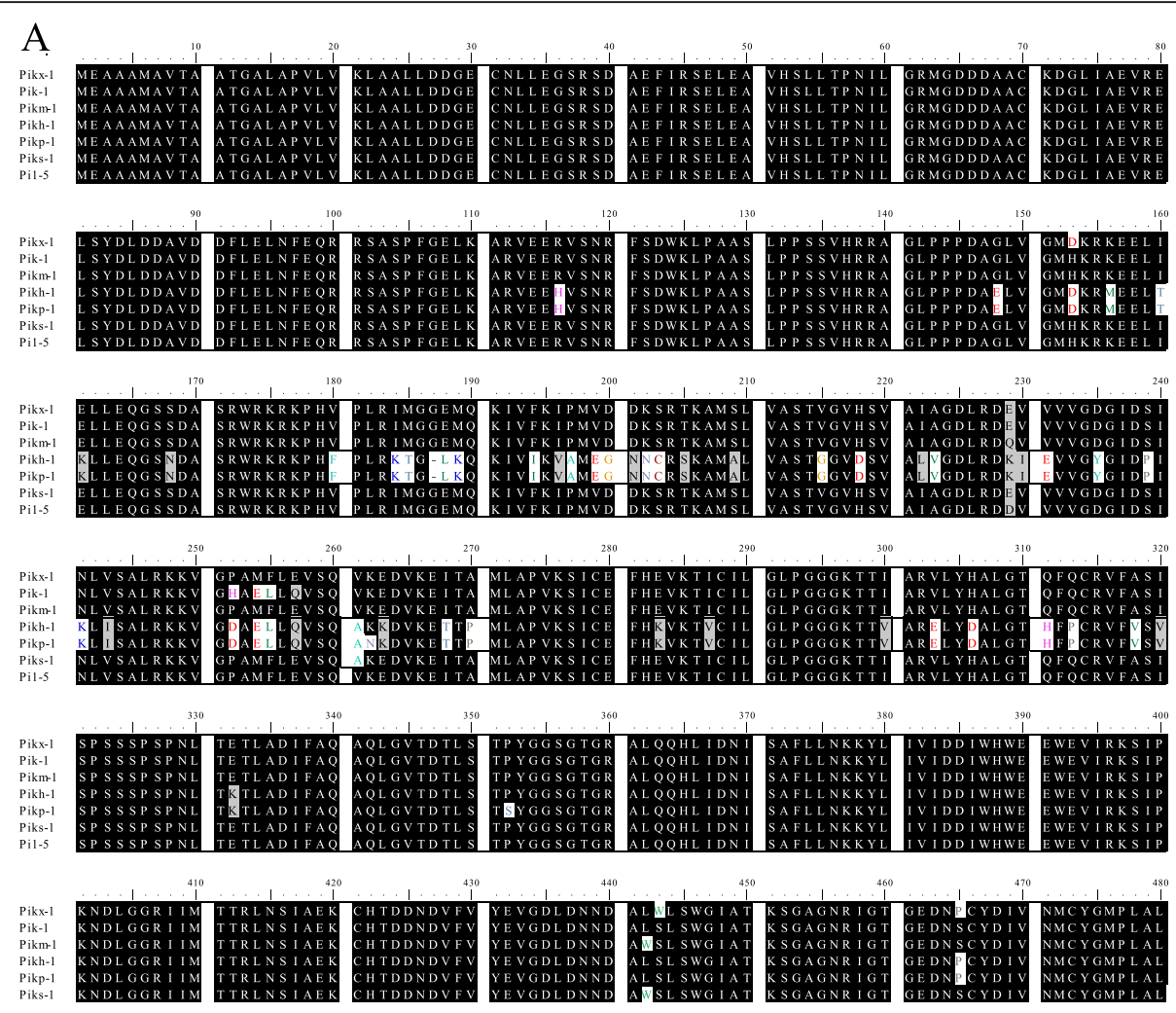

B

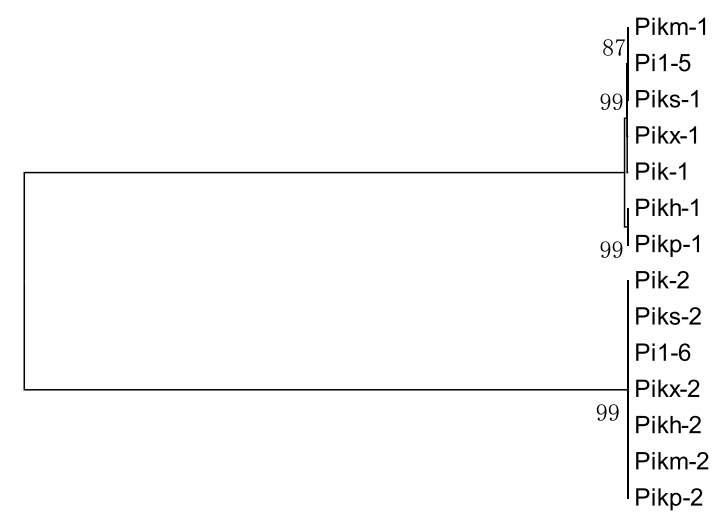

Fig. 4 Sequence comparison between Pikx and other Pik alleles a Sequence alignment of the Pik cc domain amino acid sequences using ClustalW. Shading fonts indicate common amino acid residues in the alleles. $\mathbf{b}$ Phylogenetic analysis of Pik allele protein sequences using MEGA 5. The tree was generated using UPGMA. Numbers stand for bootstrap values from 1000 replicates

(NSFTV_5, 45, 53, 93, 119, 131, 160, 321, 344, 349, and 622) by PCR amplification (Additional file 2: Table S2, highlighted with yellow). Five of them are ARO, 2 are IND, 2 are AUS and 2 are ADMIX. Among the 11 cultivars, 5 contain the AA type and 5 contain the CC type at SNP-11.27701887 (AA type is the blast resistant type as shown in Fig. 3b). Two of these cultivars, NSFTV 622 (ADMIX, AA type at SNP-11.27701887) and NSFTV_131(AUS, AA type at SNP-11.27701887), were found to harbor the Pik alleles. Sequence analysis of the predicted amino acids of the two genes indicated that an allele from cultivar NSFTV_622 was the same as the known $R$ gene $P i k$-s, and that another allele from cultivar NSFTV_131 was a new allele, which we named Pikx and which has a unique amino acid at 443S in Pikx-1 (substitution with W) (Fig. 4a). Phylogenetic analysis showed that all of the Pik allele-1 proteins are highly polymorphic but the Pik allele-2 proteins are less polymorphic (Fig. 4b). Pikx-1 was clustered in the same clade with Pikm-1, Pi1-5, Piks-1 and Pik-1 (Fig. 4b). 
A

$\mathrm{AD}$

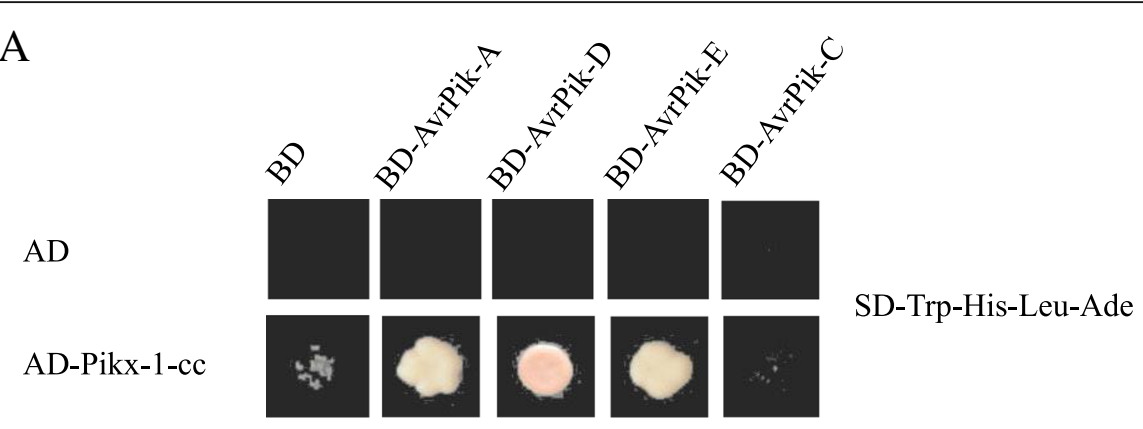

B

\begin{tabular}{|c|c|c|c|c|c|c|c|c|}
\hline \multirow[b]{2}{*}{$4 \times$ Myc-Pikx-1-cc } & \multicolumn{4}{|c|}{ Input } & \multicolumn{4}{|c|}{ IP@Myc } \\
\hline & + & + & + & + & + & + & + & + \\
\hline GFP-AvrPik-A & + & - & - & - & + & - & - & - \\
\hline GFP-AvrPik-C & - & + & - & - & - & + & - & - \\
\hline GFP-AvrPik-D & - & - & + & - & - & - & + & - \\
\hline GFP-AvrPik-E & - & - & - & + & - & - & - & + \\
\hline Anti-GFP & - & & & & $=$ & - & & \\
\hline
\end{tabular}

Fig. 5 Interaction detection between Pikx and four AvrPik proteins in yeast and in planta a Y2H analysis of the interaction between Pikx-1 cc domain and four AvrPik alleles. b Co-IP analysis of the interaction between Pikx-1 cc domain and four AvrPik alleles in N. benthamiana

A previous study reported that Pik proteins physically interact with AvrPik by the Pik-1 cc domain, and that the binding specificity between Pik-1 and AvrPik determines the recognition specificity between Pik and Avr-Pik (Kanzaki et al. 2012). To determine the relationship between Pikx and AvrPiks, we performed $\mathrm{Y} 2 \mathrm{H}$ and Co-IP assays in order to detect the interaction between Pikx and four AvrPik alleles (A, C, D, and $\mathrm{E})$. The $\mathrm{Y} 2 \mathrm{H}$ assay showed that AvrPik-A, $-\mathrm{D}$, and -E strongly interacted with Pikx-1-cc (Fig. 5a). In the Co-IP assay, however, Pikx-1-cc strongly interacted only with AvrPik-E and weakly interacted with AvrPik-A and -D (Fig. 5b). Pikm was reported to interact with AvrPik-A, $-\mathrm{C}$, and -D (Kanzaki et al. 2012). Therefore, Pikx is similar to Pikm in terms of its interaction with the AvrPik alleles.

\section{Discussion}

Given the genomic instability of $M$. oryzae populations, many resistance genes in rice are short-lived, and the identification of new blast resistance genes is therefore essential for rice breeding programs. The identification of new resistance resources can be greatly facilitated by GWAS. Phenotypic evaluations in GWAS can usually be performed within 1 year with multiple replications in the greenhouse or field, whereas conventional bi-parental QTL mapping usually requires 2 or more years for population development and phenotype evaluation. In addition, the markers used in GWAS are high throughput and high density, and the associated markers identified in the analysis can be closer to the target genes than do those identified in conventional QTL mapping. As a consequence, GWAS provides a rapid way to identify functional genes in rice. To study blast resistance in rice, Kang et al. (2016) performed GWAS of the RDP1 population and identified 97 loci associated with blast resistance, 82 of which had not been previously identified. Using GWAS in the current study, we identified 56 QTLs involved in blast resistance. Among them, 40 have not been previously identified. These results demonstrate that majority of the QTLs identified in the current study are specific to the three $M$. oryzae strains from Hunan.

Many QTLs for rice blast resistance have been previously reported (Liu et al. 2010; Liu et al. 2014). Among the loci previously identified in different mapping populations, several were also identified in our study (Deng et al. 2006; Hayashi et al. 2004; Hittalmani et al. 2000; Kang et al. 2016). For example, five 
loci were detected in the same rice population when inoculated with different blast isolates (Additional file 4: Table S4) (Kang et al. 2016). One of the five common loci is Pik on chromosome 11, which has been frequently studied in the last several years. Some of the alleles at the Pik locus such as Pik, Pik-1, Pik-p, and $P i k-m$ have been isolated and characterized (Ashikawa et al. 2008; Yuan et al. 2011; Zhai et al. 2011; Hua et al. 2012). In this study, we identified a new allele, Pikx, that is significantly associated with rice blast resistance at the Pik locus. Because it confers resistance to the three isolates collected in Hunan, we speculate that this new gene may be useful for rice breeding against the $M$. oryzae population in Hunan and other provinces in South China. The function of this gene in blast resistance will be confirmed by rice transformation in the future.

Our $\mathrm{Y} 2 \mathrm{H}$ and CoIP assays also indicated that Pikx-1 cc, which is similar to Pik-m-1-cc reported in Kanzaki et al. (2012), can interact with AvrPik-A, -D, and -E. These interactions may be possible because the key sites of the Avr-R-recognition domain HMA in the Pikx-1 cc are identical to those of the Pik-m-1 cc domain (Maqbool et al. 2015). Avr-Pik is a 113 amino acid protein with a 21 amino acid signal peptide at its $\mathrm{N}$ terminus (Yoshida et al. 2009). The latter study identified five alleles of $A v r-$ Pik (Avr-Pik-A, B, C, D, and E) in 21 isolates of M. ory$z a e$ from Japan and found that the five Avr-Pik alleles differ from one other by a total of five DNA substitutions, all of which cause amino acid changes. According to Yoshida et al. (2009), the Avr-Pik-D allele is likely the ancestral allele from which the Avr-Pik-E, $-C,-A$, and $-B$ alleles are derived. As noted earlier, Yoshida et al. (2009) found $A v r-P i k-B$ in an isolate (isolate 9505-3) from Japan. In the present study, the $\mathrm{Y} 2 \mathrm{H}$ assay showed that AvrPik-A, -D, and -E strongly interact with Pikx-1cc (Fig. 5a). In the Co-IP assay, however, Pikx-1-cc strongly interacted only with AvrPik-E and weakly interacted with AvrPik-A and -D (Fig. 5b). The reason for the difference warrants further investigation.

\section{Additional files}

Additional file 1: Table S1. Primers used in this study. (XLSX $11 \mathrm{~kb}$ ) Additional file 2: Table S2. Phenotype of 234 RDP1 varieties against Magnaporthe oryzae isolates. (XLS $61 \mathrm{~kb}$ )

Additional file 3: Table S3. The regions associated with the blast resistance QTLs to three M. oryzae isolates. (XLSX $19 \mathrm{~kb}$ )

Additional file 4: Table S4. The shared loci identified in the present study and previous study. (XLSX $13 \mathrm{~kb}$ )

\section{Acknowledgements}

We would like to thank Drs. Yuese Ning and Wende Liu for his assistance with the project

\section{Author contributions}

$\mathrm{CL}, \mathrm{DZ}, \mathrm{YL}$, and $\mathrm{G}-\mathrm{LW}$ designed and initiated this project, provided the funding, and supervised the experiments. DW, SP, YC, PS, JC, LZ, XT, JL, and $Y X$ performed experiments. $C L, D W, S P$, and G-LW analyzed the data. $C L$ and GLW wrote the manuscript. All authors have discussed the results and made comments on the manuscript. All authors have read and approved the final manuscript.

\section{Funding}

This research was supported by the National Natural Science Foundation of. China (Grant No. 31471755 to CL and Grant No. 31501628 to DW) and the. National Key Research and Development Program of China (Project No. 2016YFD0200809 to DZ).

\section{Availability of data and materials}

The datasets supporting the conclusions of this article are provided within the article and its additional files.

Ethics approval and consent to participate

Not applicable.

Consent for publication

Not applicable.

\section{Competing interests}

The authors declare that they have no competing interests.

\section{Author details}

${ }^{1}$ State Key Laboratory of Hybrid Rice and Institute of Plant Protection, Hunan Academy of Agricultural Sciences, Changsha 410125, China. ${ }^{2}$ Southern Regional Collaborative Innovation Center for Grain and Oil Crops in China and College of Agronomy, Hunan Agricultural University, Changsha 410128, Hunan, China. ${ }^{3}$ Institute of Plant Protection, Chinese Academy of Agricultural Sciences, Beijing 100193, China. ${ }^{4}$ Department of Plant Pathology, Ohio State University, Columbus, OH 43210, USA.

Received: 15 April 2019 Accepted: 30 June 2019

Published online: 15 July 2019

\section{References}

Altshuler D, Daly MJ, Lander ES (2008) Genetic mapping in human disease. science 322(5903):881-888

Ashikawa I, Hayashi N, Yamane H, Kanamori H, Wu J, Matsumoto T et al (2008) Two adjacent nucleotide-binding siteleucine rich repeat class genes are required to confer Pikm specific rice blast resistance. Genetics 180:2267-2276

Carlos DLCJ, Marina F, Abbas M, Hiromasa S, Ryohei T, Sophien K et al (2018) Polymorphic residues in rice nlrs expand binding and response to effectors of the blast pathogen. Nature Plants 4(8):576-585

Deng Y, Zhai K, Xie Z, Yang D, Zhu X, Liu J et al (2017) Epigenetic regulation of antagonistic receptors confers rice blast resistance with yield balance. Science 355(6328):962-965

Deng Y, Zhu X, Shen Y, He Z (2006) Genetic characterization and fine mapping of the blast resistance locus Pigm(t) tightly linked to Pi2 and Pi9 in a broadspectrum resistant Chinese variety. Theor Appl Genet 113:705-713

Fang N, Wang R, He W, Yin C, Guan C, Chen H et al (2016) QTL mapping of panicle blast resistance in japonica landrace heikezijing and its application in rice breeding. Mol Breed 36(12):171

Fjellstrom R, ConawayBormans CA, McClung AM, Marchetti MA, Shank AR, Park WD (2004) Development of dna markers suitable for marker assisted selection of three, genes conferring resistance to multiple, pathotypes. Cropence 44(5):1790-1798

Gnanamanickam SS (2009) Rice and its importance to human life. Biological control of rice diseases. Springer, Netherlands, pp 1-11

Hayashi K, Hashimoto N, Daigen M, Ashikawa I (2004) Development of PCR-based SNP markers for rice blast resistance genes at the Piz locus. Theor Appl Genet 108:1212-1220

Hittalmani S, Parco A, Mew TV, Zeigler RS, Huang N (2000) Fine mapping and DNA marker-assisted pyramiding of the three major genes for blast resistance in rice. Theor Appl Genet 100:1121-1128 
Hua L, Wu J, Chen C, Wu W, He X, Lin F et al (2012) The isolation of Pi1, an allele at the Pik locus which confers broad spectrum resistance to rice blast. Theor Appl Genet 125:1047-1055

Huang X, Wei X, Sang T, Zhao Q, Feng Q, Zhao Y et al (2010) Genome-wide association studies of 14 agronomic traits in rice landraces. Nat Genet 42(11):961-967

Hulbert SH, Webb CA, Smith SM, Sun Q (2001) Resistance gene complexes: evolution and utilization. Annu Rev Phytopathol 39:285-312

Kaler AS, Ray JD, Schapaugh WT, King CA, Purcell LC (2017) Genome-wide association mapping of canopy wilting in diverse soybean genotypes. Theor Appl Genet 130:2203

Kang H, Wang Y, Peng S, Zhang Y, Xiao Y, Wang D et al (2016) Dissection of the genetic architecture of rice resistance to the blast fungus Magnaporthe oryzae. Mol Plant Pathol 17(6):959-972

Kang HM, Sul JH, Service, S. K, Zaitlen NA, Kong SY, Freimer NB et al (2010) Variance component model to account for sample structure in genomewide association studies. Nat Genet 42(4):348-354

Kanzaki H, Yoshida K, Saitoh H, Fujisaki K, Hirabuchi A, Alaux L et al (2012) Arms race co-evolution of Magnaporthe oryzae AVR-Pik and rice Pik genes driven by their physical interactions. Plant J 72(6):894-907

Li W, Zhu Z, Chern M, Yin J, Yang C, Ran L, et al. (2017) A natural allele of a transcription factor in rice confers broad-spectrum blast resistance. Cell 170(1):114-126.e15

Liu J, Wang X, Mitchell T, Hu Y, Liu X, Dai L, Wang GL (2010) Recent progress and understanding of the molecular mechanisms of the rice-Magnaporthe oryzae interaction. Mol Plant Pathol 11(3):419-427

Liu WD, Liu JL, Leach JE, Wang GL (2014) Novel insights into rice innate immunity against bacterial and fungal pathogens. Annu Rev Phytopathol 52:213-241

Liu X, Huang M, Fan B, Buckler ES, Zhang Z (2016) Iterative usage of fixed and random effect models for powerful and efficient genome-wide association studies. PLoS Genet 12(2):e1005767

Lv Y, Guo Z, Li X, Ye H, Li X, Xiong L (2016) New insights into the genetic basis of natural chilling and cold shock tolerance in rice by genome-wide association analysis. Plant Cell Environ 39(3):556-570. https://doi.org/10.1111/ pce. 12635

Maqbool A, Saitoh H, Franceschetti M, Stevenson CE, Uemura A, Kanzaki H et al (2015) Structural basis of pathogen recognition by an integrated HMA domain in a plant NLR immune receptor. Elife 4:e08709. https://doi.org/10. 7554/eLife.08709

Mgonja EM, Balimponya EG, Kang H, Bellizzi M, Park CH, Li Y et al (2016) Genome-wide association mapping of Rice resistance genes against Magnaporthe oryzae isolates from four African countries. Phytopathology. 106(11):1359-1365

Oliveira-Garcia E, Valent B (2015) How eukaryotic filamentous pathogens evade plant recognition. Curr Opin Microbiol 26:92-101

Park CH, Chen S, Shirsekar G, Zhou B, Khang CH, Songkumarn P et al (2012) The Magnaporthe oryzae effector AvrPiz-t targets the RING E3 ubiquitin ligase APIP6 to suppress pathogen-associated molecular pattern-triggered immunity in rice. Plant Cell 24(11):4748-4762

Price AL, Patterson NJ, Plenge RM, Weinblatt ME, Shadick NA, Reich D (2006) Principal components analysis corrects for stratification in genome-wide association studies. Nat Genet 38:904-909

Si L, Chen J, Huang X, Gong H, Luo J, Hou Q et al (2016) OsSPL13 controls grain size in cultivated rice. Nat Genet 48(4):447-456

Skamnioti P, Gurr SJ (2009) Against the grain: safeguarding rice from rice blast disease. Trends Biotechnol 27(3):141-150

Valent B, Chumley FG (1991) Molecular genetic analysis of the rice blast fungus, Magnaporthe grisea. Annu Rev Phytopathol 29:443-467

Wang C, Yang Y, Yuan X, Xu Q, Feng Y, Yu H et al (2014) Genome-wide association study of blast resistance in indica rice. BMC Plant Biol 18(14):311

Wang D, Liu J, Li C, Kang H, Wang Y, Tan X et al (2016a) Genome-wide association mapping of cold tolerance genes at the seedling stage in rice. Rice 9:61

Wang R, Ning Y, Shi X, He F, Zhang C, Fan J et al (2016b) Immunity to Rice Blast Disease by Suppression of Effector-Triggered Necrosis. Curr Biol 26(18):2399-2411

Wu W, Wang L, Zhang S, Li Z, Zhang Y, Lin F et al (2014) Stepwise arms race between AvrPik and Pik alleles in the rice blast pathosystem. Mol PlantMicrobe Interact 27(8):759-769

Xiao N, Wu Y, Pan C, Yu L, Chen Y, Liu G, et al (2017) Improving of rice blast resistances in japonica by pyramiding major $r$ genes. Frontiers in Plant Science, 7
Yang QZ, Lin F, Feng SJ, Wang L, Pan QH (2009) Recent progress on molecular mapping and cloning of blast resistance genes in rice (Oryza sativa L.). Sci Agric Sin 42:1601-1615

Yano K, Yamamoto E, Aya K, Takeuchi H, Lo PC, Hu L et al (2016) Genome-wide association study using whole-genome sequencing rapidly identifies new genes influencing agronomic traits in rice. Nat Genet 48(8):927-934

Yoshida K, Saitoh H, Fujisawa S, Kanzaki H, Matsumura H, Yoshida K et al (2009) Association genetics reveals three novel avirulence genes from the rice blast fungal pathogen Magnaporthe oryzae. Plant Cell 21(5):1573-1591

Yu J, Pressoir G, Briggs WH, Vroh Bi I, Yamasaki M, Doebley JF et al (2006) A unified mixed-model method for association mapping that accounts for multiple levels of relatedness. Nat Genet 38:203-208

Yuan B, Zhai C, Wang W, Zeng X, Xu X, Hu H et al (2011) The Pik-p resistance to Magnaporthe oryzae in rice is mediated by a pair of closely linked CC-NBSLRR genes. Theor Appl Genet 122:1017-1028

Zhai C, Lin F, Dong Z, He X, Yuan B, Zeng X et al (2011) The isolation and characterization of Pik, a rice blast resistance gene which emerged after rice domestication. New Phytol 189:321-334

Zhao H, Wang X, Jia Y, Minkenberg B, Yang Y (2018) The rice blast resistance gene ptr encodes an atypical protein required for broad-spectrum disease resistance. Nat Commun 9(1):2039

Zhao K, Tung CW, Eizenga GC, Wright MH, Ali ML, Price AH et al (2011) Genomewide association mapping reveals a rich genetic architecture of complex traits in Oryza sativa. Nat Commun 2:467

Zhu D, Kang H, Li Z, Liu M, Zhu X, Wang Y et al (2016) A genome-wide association study of field resistance to Magnaporthe Oryzae in Rice. Rice (N Y) 9(1):44

Zhu Y, Chen K, Mi X, Chen T, Ali J, Ye G et al (2015) Identification and fine mapping of a stably expressed QTL for cold tolerance at the booting stage using an interconnected breeding population in Rice. PLoS One 10(12): e0145704

\section{Publisher's Note}

Springer Nature remains neutral with regard to jurisdictional claims in published maps and institutional affiliations.

\section{Submit your manuscript to a SpringerOpen ${ }^{\circ}$ journal and benefit from:}

- Convenient online submission

- Rigorous peer review

- Open access: articles freely available online

- High visibility within the field

- Retaining the copyright to your article

Submit your next manuscript at $\boldsymbol{\nabla}$ springeropen.com 